\title{
A NUMERICAL EXAMPLE FOR THE INTERSECTION OF THE COMPATIBLE QUASILINEAR EXTENSIONS OF A PARTIAL ORDER
}

\author{
ATTILA HÁZY, PÉTER OLAJOS, IMRE PILLER, AND SZILVIA SZILÁGYI \\ Received 20 December, 2017
}

\begin{abstract}
We present a numerical example for the intersection of the maximal compatible extensions of a partial order.
\end{abstract}

2010 Mathematics Subject Classification: 06A06; 06F99

Keywords: compatible partial order, cyclic element, quasilinear partial order

\section{INTRODUCTION}

One of the typical problems in the theory of ordered algebras is to describe the intersection of the compatible linear extensions of $\leq_{r}$ in a partially ordered algebraic structure $\left(A, F, \leq_{r}\right)$. If $F=\varnothing$, then the answer can be found in [10]. Szpilrajn proved that any partial order $\leq_{r}$ (or $r$ ) on a set $A$ can be extended to a linear order $\leq_{R}$ (or $R$ ). He also proved that any partial order is the intersection of its linear extensions. For $F=\{f\}$, where $f: A \rightarrow A$ is a unary operation with the natural compatibility property ( $x \leq_{r} y$ implies $f(x) \leq_{r} f(y)$ for all $x, y \in A$ ) the above problem has been investigated. Szigeti and Nagy proved that the partially ordered mono-unary algebra $\left(A, f, \leq_{r}\right)$ has a compatible linear extension if and only if $f$ is acyclic (see [8]). For an acyclic partially ordered unary algebra $\left(A, f, \leq_{r}\right)$ the intersection of the compatible linear extensions of $r$ is determined in [7]. Foldes and Szigeti in [6] proved that any compatible partial order on the unary algebra $(A, f)$ can be extended to a so called compatible $f$-quasilinear partial order. A consequence of this fact is that the maximal compatible partial orders are exactly the compatible $f$ quasilinear partial orders on $(A, f)$. If the function $f$ has no proper cycle (acyclic), then a compatible partial order is $f$-quasilinear if and only if it is linear. For an acyclic partially ordered unary algebra $\left(A, f, \leq_{r}\right)$ the intersection of the compatible linear extensions of $r$ is determined in [7]. A new characterization of the maximal compatible extensions of $r$ and the description of the intersection of these extensions in an arbitrary (possibly non-acyclic) triple $\left(A, f, \leq_{r}\right)$ can be seen in [9]. 
In the first part of our paper we investigate a finite variant of the example for the mono-unary partially ordered algebra in [9]. The second part of our paper we use computer calculations to provide the explicit description of the intersection in the above mentioned finite example.

In order to present a self contained treatment, in section 2 we provide the necessary prerequisites.

\section{PRELIMINARY DEFINITIONS AND RESUlTS}

Let $(A, f)$ be a unary algebra. A partial order $\leq_{r}$ on $A$ is called compatible on $(A, f)$ if the function (unary operation) $f: A \longrightarrow A$ is an order endomorphism with respect to $\leq_{r}\left(x \leq_{r} y\right.$ implies $f(x) \leq_{r} f(y)$ for all $\left.x, y \in A\right)$. The equivalence relation $\sim_{f}$ is defined in [9] as follows: for $x, y \in A$ let $x \sim_{f} y$ if $f^{k}(x)=f^{l}(y)$ for some integers $k \geq 0$ and $l \geq 0$. The equivalence class $[x]_{f}$ of an element $x \in A$ is called the $f$-component of $x$. Clearly, $[x]_{f}$ contains the $f$-orbit of $x$ :

$$
\left\{x, f(x), \ldots, f^{k}(x), \ldots\right\} \subseteq[x]_{f} .
$$

An element $c \in A$ is called cyclic with respect to $f$, if $f^{m}(c)=c$ for some integer $m \geq 1$. The period of a cyclic element $c$ is

$$
n=n(c)=\min \left\{m \mid m \geq 1 \text { and } f^{m}(c)=c\right\}
$$

and $f^{k}(c)=f^{l}(c)$ holds if and only if $k-l$ is divisible by $n$. The cycle $C=$ $\left\{c, f(c), \ldots, f^{n-1}(c)\right\}$ has exactly $n$ elements and $f(C)=C$. The $f$-orbit of $x$ is finite if and only if $[x]_{f}$ contains a cyclic element. The function $f$ has a proper cycle if there exists a cyclic element $c \in A$ with respect to $f$ such that $n(c) \geq 2$. When $n(c)=1$ then $f(c)=c$. In this case $c \in A$ is a fixed point of $f$. The concept of the $f$-prohibited pair was introduced by Foldes and Szigeti in [6]. A pair $(x, y) \in A \times A$ is called $f$-prohibited, if we can find integers $k \geq 0, l \geq 0$ and $n \geq 2$ such that $n$ is not a divisor of $k-l$, the elements $f^{k}(x), f^{k+1}(x), \ldots, f^{k+n-1}(x)$ are distinct and $f^{k+n}(x)=f^{k}(x)=f^{l}(y)$. For an $f$-prohibited pair $(x, y)$ and for the integers $k \geq 0, n \geq 2$ as above, $f^{k}(x)$ is a cyclic element in $[x]_{f}=[y]_{f}$ of period $n$.

Proposition 1 (see [6]). If $\left(A, f, \leq_{r}\right)$ is a partially ordered unary algebra, then we have the following.

(1) If $(x, y)$ is $f$-prohibited, then $(y, x)$ is also $f$-prohibited.

(2) If $(x, y)$ is not $f$-prohibited, then $(f(x), f(y))$ is also not $f$-prohibited.

(3) If $[x]_{f} \neq[y]_{f}$, then $(x, y)$ is not $f$-prohibited.

(4) If the $f$-orbit of $x$ is infinite, then there is no cyclic element in $[x]_{f}$ and hence $(x, y)$ is not $f$-prohibited (even in the case of $[x]_{f}=[y]_{f}$ ).

(5) If $(x, y) \in A \times A$ is an $f$-prohibited pair, then $x$ and $y$ are incomparable with respect to $\leq_{r}$ (in particular, any two distinct elements in a full cycle are incomparable with respect to $\leq_{r}$ ). 
A compatible partial order $R$ on a unary algebra $(A, f)$ is called $f$-quasilinear, if $(x, y) \in R$ or $(y, x) \in R$ for all non $f$-prohibited pairs $(x, y) \in A \times A$. We use of the following notations of [9]:

$\mathcal{Q L}\left(A, f, \leq_{r}\right)=\{R \mid r \subseteq R \subseteq A \times A$ is a compatible $f$-quasilinear partial order $\}$,

$\mathscr{L}\left(A, f, \leq_{r}\right)=\{R \mid r \subseteq R \subseteq A \times A$ is a compatible linear order $\}$.

The intersection

$$
\operatorname{cl}\left(A, f, \leq_{r}\right)=\bigcap_{R \in \mathcal{Q} \mathscr{L}(A, f, \leq r)} R
$$

is called the closure of $r$ with respect to $(A, f)$. Since $\mathcal{Q} \mathscr{L}\left(A, f, \leq_{r}\right)$ is never empty by the Main Theorem of [6], the above definition gives a closure operator (with the monotone, idempotent and extensive properties) on the set of the compatible partial orders of $(A, f)$.

An element $a \in A$ of the partially ordered unary algebra $\left(A, f, \leq_{r}\right)$ is

(1) $\uparrow$-definite if $f^{p}(a)<_{r} f^{q}(a)$ for some integers $0 \leq p<q$,

(2) $\downarrow$-definite if $f^{q}(a)<_{r} f^{p}(a)$ for some integers $0 \leq p<q$,

(3) indefinite if $f^{p}(a)$ and $f^{q}(a)$ are $r$-incomparable for all integers $0 \leq p, q$ with $f^{p}(a) \neq f^{q}(a)$.

It can be easily checked if $\left(A, f, \leq_{r}\right)$ is a partially ordered unary algebra and $a \in A$, then $a$ satisfies exactly one of the above $\uparrow$-definite, $\downarrow$-definite and indefinite properties. Define the sets $M_{\uparrow}, M_{\downarrow}$ and $M$ as in [7].

$$
\begin{gathered}
M_{\uparrow}=\{(x, y) \in A \times A \mid x \text { is } \uparrow \text {-definite, }(\exists m)(\exists t) 0 \leq m \leq t, \\
\left.\quad f^{t}(x) \leq r f^{m}(y) \neq f^{m}(x)\right\}, \\
M_{\downarrow}=\{(x, y) \in A \times A \mid x \text { is } \downarrow \text {-definite, }(\exists m)(\exists t) 0 \leq t \leq m, \\
\left.f^{t}(x) \leq r f^{m}(y) \neq f^{m}(x)\right\}, \\
M=\left\{(x, y) \in A \times A \mid x \text { is indefinite, }(\exists m)(\exists t)\left(\exists m^{\prime}\right)\left(\exists t^{\prime}\right) 0 \leq m \leq t, 0 \leq t^{\prime} \leq m^{\prime},\right. \\
\left.f^{t}(x) \leq r f^{m}(y) \neq f^{m}(x), f^{t^{\prime}}(x) \leq r f^{m^{\prime}}(y) \neq f^{m^{\prime}}(x)\right\} .
\end{gathered}
$$

We shall make use of the set

$$
P=\{(x, y) \in A \times A \mid(x, y) \text { is not } f \text {-prohibited }\} .
$$

One of the main theorems of [9] gives a complete description of the intersection of the maximal compatible extensions of $r$ :

Theorem 1. If $\left(A, f, \leq_{r}\right)$ is a partially ordered unary algebra, then

$$
\boldsymbol{c l}\left(A, f, \leq_{r}\right)=\left(M_{\uparrow} \cup M_{\downarrow} \cup M \cup\{(x, x) \mid x \in A\}\right) \cap P .
$$




\section{A NUMERICAL EXAMPLE}

The following example can be found in [9]. Let

$$
2 \leq p_{1}<p_{2}<p_{3}<q_{1}<q_{2}<q_{3}<q_{4}<r
$$

be a sequence of prime numbers and consider the infinite set

$$
\begin{gathered}
A=\left\{p_{1}^{m_{1}} p_{2}^{m_{2}} p_{3}^{m_{3}} q_{1}^{n_{1}} q_{2}^{n_{2}} q_{3}^{n_{3}} q_{4}^{n_{4}} r^{k} \mid\right. \\
\left.m_{i} \geq 0, n_{j} \geq 0, i \in\{1,2,3\}, j \in\{1,2,3,4\}, k \geq 0\right\}
\end{gathered}
$$

of integers. A natural partial order on $A$ is the divisibility relation: for $x, y \in A$ we use the notation $x \leq_{d} y$ if $x \mid y$.

Define the function $f: A \rightarrow A$ on $x=p_{1}^{m_{1}} p_{2}^{m_{2}} p_{3}^{m_{3}} q_{1}^{n_{1}} q_{2}^{n_{2}} q_{3}^{n_{3}} q_{4}^{n_{4}} r^{k}$ by

$$
f\left(p_{1}^{m_{1}} p_{2}^{m_{2}} p_{3}^{m_{3}} q_{1}^{n_{1}} q_{2}^{n_{2}} q_{3}^{n_{3}} q_{4}^{n_{4}} r^{k}\right)=p_{1}^{m_{3}} p_{2}^{m_{1}} p_{3}^{m_{2}} q_{1}^{n_{1}+n_{2}} q_{2}^{n_{3}} q_{3}^{n_{4}} r^{\langle k-1\rangle},
$$

where $\langle l\rangle=l$ if $l \geq 0$ and $\langle l\rangle=0$ if $l<0$. Note that $\langle\langle l\rangle-1\rangle=\langle l-1\rangle$. Clearly, $x \leq_{d} y$ implies that $f(x) \leq_{d} f(y)$. We shall make use of the following calculations:

$$
\begin{aligned}
& f^{2}\left(p_{1}^{m_{1}} p_{2}^{m_{2}} p_{3}^{m_{3}} q_{1}^{n_{1}} q_{2}^{n_{2}} q_{3}^{n_{3}} q_{4}^{n_{4}} r^{k}\right)=p_{1}^{m_{2}} p_{2}^{m_{3}} p_{3}^{m_{1}} q_{1}^{n_{1}+n_{2}+n_{3}} q_{2}^{n_{4}} r^{\langle k-2\rangle}, \\
& f^{3}\left(p_{1}^{m_{1}} p_{2}^{m_{2}} p_{3}^{m_{3}} q_{1}^{n_{1}} q_{2}^{n_{2}} q_{3}^{n_{3}} q_{4}^{n_{4}} r^{k}\right)=p_{1}^{m_{1}} p_{2}^{m_{2}} p_{3}^{m_{3}} q_{1}^{n_{1}+n_{2}+n_{3}+n_{4}} r^{\langle k-3\rangle}, \\
& f^{4}\left(p_{1}^{m_{1}} p_{2}^{m_{2}} p_{3}^{m_{3}} q_{1}^{n_{1}} q_{2}^{n_{2}} q_{3}^{n_{3}} q_{4}^{n_{4}} r^{k}\right)=p_{1}^{m_{3}} p_{2}^{m_{1}} p_{3}^{m_{2}} q_{1}^{n_{1}+n_{2}+n_{3}+n_{4}} r^{\langle k-4\rangle,} \\
& f^{5}\left(p_{1}^{m_{1}} p_{2}^{m_{2}} p_{3}^{m_{3}} q_{1}^{n_{1}} q_{2}^{n_{2}} q_{3}^{n_{3}} q_{4}^{n_{4}} r^{k}\right)=p_{1}^{m_{2}} p_{2}^{m_{3}} p_{3}^{m_{1}} q_{1}^{n_{1}+n_{2}+n_{3}+n_{4}} r^{\langle k-5\rangle}, \\
& f^{6}\left(p_{1}^{m_{1}} p_{2}^{m_{2}} p_{3}^{m_{3}} q_{1}^{n_{1}} q_{2}^{n_{2}} q_{3}^{n_{3}} q_{4}^{n_{4}} r^{k}\right)=p_{1}^{m_{1}} p_{2}^{m_{2}} p_{3}^{m_{3}} q_{1}^{n_{1}+n_{2}+n_{3}+n_{4}} r^{\langle k-6\rangle} .
\end{aligned}
$$

It follows that $f^{6}(x) \leq_{d} f^{3}(x)$ and $f^{\langle k-3\rangle+6}(x)=f^{\langle k-3\rangle+3}(x)$ for all $x \in A$. Thus $f^{6}(x) \neq f^{3}(x)$ implies that $x$ is $\downarrow$-definite. Obviously, $f^{6}(x)=f^{3}(x)$ holds if and only if $0 \leq k \leq 3$. For all $x \in A$ and $i \in\{1,2,4,5\}$ the following equivalences are straightforward consequences of the above calculations:

(P1) $x \leq_{d} f^{i}(x) \Longleftrightarrow m_{1}=m_{2}=m_{3}, n_{2}=n_{3}=n_{4}=k=0$ and $x=f(x)$,

(P2) $x \leq_{d} f^{3}(x) \Longleftrightarrow n_{2}=n_{3}=n_{4}=k=0$ and $x=f^{3}(x)$,

(P3) $f^{i}(x) \leq_{d} x \Longleftrightarrow m_{1}=m_{2}=m_{3}, n_{2}=n_{3}=n_{4}=0$ and $x=f(x) r^{k-\langle k-1\rangle}$,

(P4) $f^{3}(x) \leq_{d} x \Longleftrightarrow n_{2}=n_{3}=n_{4}=0$ and $x=f^{3}(x) r^{k-\langle k-3\rangle}$.

There is no $\uparrow$-definite element in the set $\mathrm{A}$, so the set $M_{\uparrow}$ is empty. The element $x$ is indefinite if and only if one of the following conditions holds:

(Q1) $k=0$,

(Q2) $k=1$ and $\left\{n_{2}, n_{3}, n_{4}\right\} \neq\{0\}$,

(Q3) $k=2$ and $\left\{n_{3}, n_{4}\right\} \neq\{0\}$,

(Q4) $k=3$ and $n_{4} \neq 0$. 
In all other cases $x$ is $\downarrow$-definite.

Take

$x_{1}=p_{1}^{1} p_{2}^{2} p_{3}^{3} q_{1} q_{2} q_{3} q_{4} r^{5}, x_{2}=f^{5}\left(x_{1}\right)=p_{1}^{3} p_{2}^{1} p_{3}^{2} q_{1}^{4}$ and $y=p_{1}^{2} p_{2}^{3} p_{3}^{4} q_{1} q_{2} q_{3} q_{4} r^{4}$. Now $\left[x_{1}\right]_{f}=\left[x_{2}\right]_{f} \neq[y]_{f}$ ensures that $\left(x_{1}, y\right)$ and $\left(x_{2}, y\right)$ are non $f$-prohibited pairs. Since $f^{6}\left(x_{1}\right)<_{d} f^{3}\left(x_{1}\right)$ and $\left\{x_{2}, f\left(x_{2}\right), f^{2}\left(x_{2}\right)\right\}$ is an antichain, we obtain that $x_{1}$ is $\downarrow$-definite and $x_{2}$ is indefinite. The relations

$$
\begin{gathered}
f^{5}\left(x_{1}\right) \leq_{d} f^{7}(y) \neq f^{7}\left(x_{1}\right) \text { and } f^{0}\left(x_{2}\right) \leq_{d} f^{7}(y) \neq f^{7}\left(x_{2}\right), \\
f^{6}\left(x_{2}\right) \leq_{d} f^{4}(y) \neq f^{4}\left(x_{2}\right)
\end{gathered}
$$

imply that $\left(x_{1}, y\right) \in M_{\downarrow} \cap P$ and $\left(x_{2}, y\right) \in M \cap P$. Observe that $\left(x_{1}, y\right)$ and $\left(x_{2}, y\right)$ are incomparable pairs with respect to $\leq_{d}$ and

$$
\left(x_{1}, y\right),\left(x_{2}, y\right) \in\left(M_{\downarrow} \cup M \cup\{(x, x) \mid x \in A\}\right) \cap P=\operatorname{cl}\left(A, f, \leq_{d}\right)
$$

by Theorem 2.2. The full description of $M_{\downarrow}$ and $M$ would require a lengthy examination.

Now we consider the following finite subset of $A$ :

$$
\begin{gathered}
B=\left\{2^{m_{1}} 3^{m_{2}} 5^{m_{3}} 7^{n_{1}} 11^{n_{2}} 13^{n_{3}} 17^{n_{4}} 19^{k}\right. \\
\left.0 \leq m_{i}, n_{j}, k \leq K, i \in\{1,2,3\}, j \in\{1,2,3,4\}\right\} .
\end{gathered}
$$

The pair $\left(B, \leq_{d}\right)$ is a partially ordered set, where $\leq_{d}$ is the divisibility relation as above. The function $g: B \rightarrow B$ on $x \in B$ as follows:

$$
\begin{aligned}
g(x) & =g\left(2^{m_{1}} 3^{m_{2}} 5^{m_{3}} 7^{n_{1}} 11^{n_{2}} 13^{n_{3}} 17^{n_{4}} 19^{k}\right) \\
& =2^{m_{3}} 3^{m_{1}} 5^{m_{2}} 7^{n_{1}+n_{2}} 11^{n_{3}} 13^{n_{4}} 19^{\langle k-1\rangle},
\end{aligned}
$$

where

$$
\rangle t\left\langle= \begin{cases}t, & \text { if } t \leq K \\ K, & \text { if } t>K\end{cases}\right.
$$

and

$$
\langle l\rangle= \begin{cases}l, & \text { if } l \geq 0, \\ 0, & \text { if } l<0 .\end{cases}
$$

Note that $\rangle\rangle t\langle+s\langle=\rangle t+s\langle$ and $\langle\langle l\rangle-1\rangle=\langle l-1\rangle$ for all positive integers.

The function $g$ has the natural compatibility property: $x \leq_{d} y$ implies $g(x) \leq_{d}$ $g(y)$ for all $x, y \in B$. For the iterates of $g$ we have:

$$
\begin{aligned}
& g^{2}(x)=2^{m_{2}} 3^{m_{3}} 5^{m_{1}} 7^{n_{1}+n_{2}+n_{3} \backslash} 11^{n_{4}} 19^{\langle k-2\rangle}, \\
& g^{3}(x)=2^{m_{1}} 3^{m_{2}} 5^{m_{3}} 7^{\dagger n_{1}+n_{2}+n_{3}+n_{4} \backslash} 19^{\langle k-3\rangle}, \\
& g^{4}(x)=2^{m_{3}} 3^{m_{1}} 5^{m_{2}} 7^{\eta_{1}+n_{2}+n_{3}+n_{4} \backslash} 19^{\langle k-4\rangle}, \\
& g^{5}(x)=2^{m_{2}} 3^{m_{3}} 5^{m_{1}} 7^{\rangle n_{1}+n_{2}+n_{3}+n_{4} \backslash} 19^{\langle k-5\rangle}, \\
& g^{6}(x)=2^{m_{1}} 3^{m_{2}} 5^{m_{3}} 7^{\eta_{1}+n_{2}+n_{3}+n_{4} \backslash} 19^{\langle k-6\rangle} .
\end{aligned}
$$


$x \in B$ is a fixed point of $g$ if

$$
\begin{aligned}
x & =2^{m_{1}} 3^{m_{2}} 5^{m_{3}} 7^{n_{1}} 11^{n_{2}} 13^{n_{3}} 17^{n_{4}} 19^{k} \\
& =2^{m_{3}} 3^{m_{1}} 5^{m_{2}} 7^{\rangle n_{1}+n_{2}} 11^{n_{3}} 13^{n_{4}} 19^{\langle k-1\rangle}=g(x) .
\end{aligned}
$$

The above equality holds if and only if $m_{1}=m_{2}=m_{3}, n_{2}=n_{3}=n_{4}=k=0$ and $m_{1}, n_{1} \in\{0,1, \ldots, K\}$, therefore the number of fixed points is $(K+1)^{2}$. If the exponent of every prime number is at most 1 , then $|B|=2^{8}=256$ and the function $g$ has four fixed points:

(a) $m_{1}=m_{2}=m_{3}=0$ and $n_{1}=0$, then $x_{1}=1$;

(b) $m_{1}=m_{2}=m_{3}=0$ and $n_{1}=1$, then $x_{2}=7$;

(c) $m_{1}=m_{2}=m_{3}=1$ and $n_{1}=0$, then $x_{3}=30$;

(d) $m_{1}=m_{2}=m_{3}=1$ and $n_{1}=1$, then $x_{4}=210$.

Obviously, the fixed points of $g$ are in different $g$-components. If $K=1$ there it is easy to see that:

$$
[1]_{g}=\{1,19\} \quad \text { and } \quad[30]_{g}=\{30,570\} .
$$

The $g$-components of the other two fixed points contain more elements. If $x \in B$ is a fixed point, then the equivalence class $[x]_{g}$ has no proper cyclic elements. Clearly, any fixed point of $g$ is an indefinite element in the partially ordered unary algebra $\left(B, g, \leq_{d}\right)$.

The proper cyclic elements can be obtained from the properties (P2) and (P4). If $K=1$, it is deemed to be sufficient for the property (P2). $c \in A$ is a cyclic element of $g$, according to the property (P2), when $c=g^{3}(c)$, i. e. $n_{2}=n_{3}=n_{4}=k=0$ and $m_{i}, n_{1} \in\{0,1\}, i \in\{1,2,3\}$, furthermore $m_{1} \neq m_{3}$. We have four proper cycle:

$$
C_{1}=\{2,3,5\} ; \quad C_{2}=\{6,10,15\} ; \quad C_{3}=\{14,21,35\} ; \quad C_{4}=\{42,70,105\} .
$$

We note that $[2]_{g},[6]_{g},[14]_{g}$ and $[42]_{g}$ are disjoint $g$-components of $\left(B, g, \leq_{d}\right)$. It is clear, that there is no $\uparrow$-definite element in the set $B$ and the set $M_{\uparrow}$ is empty as in the above infinite example.

As we can see it above, the calculation of the elements of the intersection is time consuming manually. These particular calculations do not provide us to see the underlying structures.

\section{Calculating intersections by Maple, Magma and a Parallel PROGRAM}

For calculating the pairs of intersection we had three programs. At first we calculated them for $K=1$ by Maple, but it was very slow and a lot of resources were used.

Secondary we made it by MAGMA [3] which was stable and produced the correct results. E.g. MAGMA used 157 seconds only in CPU time. The version of MAGMA was 2.18-4. 
The third one was a parallel program which was made in JCluster [2]. It is an environment using JAVA to make and run parallel algorithms (programs) on more then one workstations. This program used very simple technics e.g. exchange a lot of "for" cycles by parellel processes. To reduce the CPU time we used 1, 2, 4, 8 and 16 workstations like a super PC with $1 \mathrm{~GB}$ network. Beacuse of it we have to mention that in the case when large number of workstations or cores were used the CPU time has increased by the network communication delay and the data broadcasting (see the table below). Certainly this program used synchronization tools also to get correct results.

The running times (in CPU time) collected in the following table:

\begin{tabular}{|c|c|c|}
\hline Environment & CPU time (sec) & Number of cores \\
\hline Maple & 425 & 4 \\
\hline MAGMA & 157 & 4 \\
\hline Parallel program (JCluster) & 67 & 1 \\
\hline Parallel program (JCluster) & 55 & 2 \\
\hline Parallel program (JCluster) & 42 & 4 \\
\hline Parallel program (JCluster) & 35 & 8 \\
\hline
\end{tabular}

\section{Calculation of the Finite example}

The actual implementation of the calculation is primarily based on the previous Maple and Magma scripts. All implementations provide the same results but the new $\mathrm{C}++$ version run more efficiently. We can see the dataflow on Figure 1.

The prims is the array of prime numbers. In this case it contains only eight elements.

$$
\text { prims }=[2,3,5,7,11,13,17,19]
$$

In the definition of the set $B$ the exponents of the factors must be non-negative integers. The $K$ value is the upper bound of them:

$$
0 \leq m_{i}, n_{j}, k \leq K, \quad \forall i \in\{1,2,3\}, \quad j \in\{1,2,3,4\} .
$$

We can consider the exponents of the factorization of the elements of $B$ as the digits of a number in $K+1$ based numerical system. From this reason $|B|=(K+1)^{8}$. In most cases in the implementation we use lists instead of sets. The lists are indexed by non-negative integers. The first index is the 0 . The index of any $B$ element is the value of the exponents of prime factorization in the mentioned $K+1$ based numerical system. For example when $K=1$ then $30=2^{1} \cdot 3^{1} \cdot 5^{1} \cdot 7^{0} \cdot 11^{0} \cdot 13^{0} \cdot 17^{0} \cdot 19^{0}$, the index is 7 . Let denote indices a function which calculate this index from elements of $B$.

We need to calculate often with powers of $g$ function. The $\mathrm{CP} 0$ contains the indices of following elements in $\mathrm{B}$ :

$$
\left[x, g(x), g^{2}(x), g^{3}(x), g^{4}(x), g^{5}(x), g^{6}(x)\right],
$$




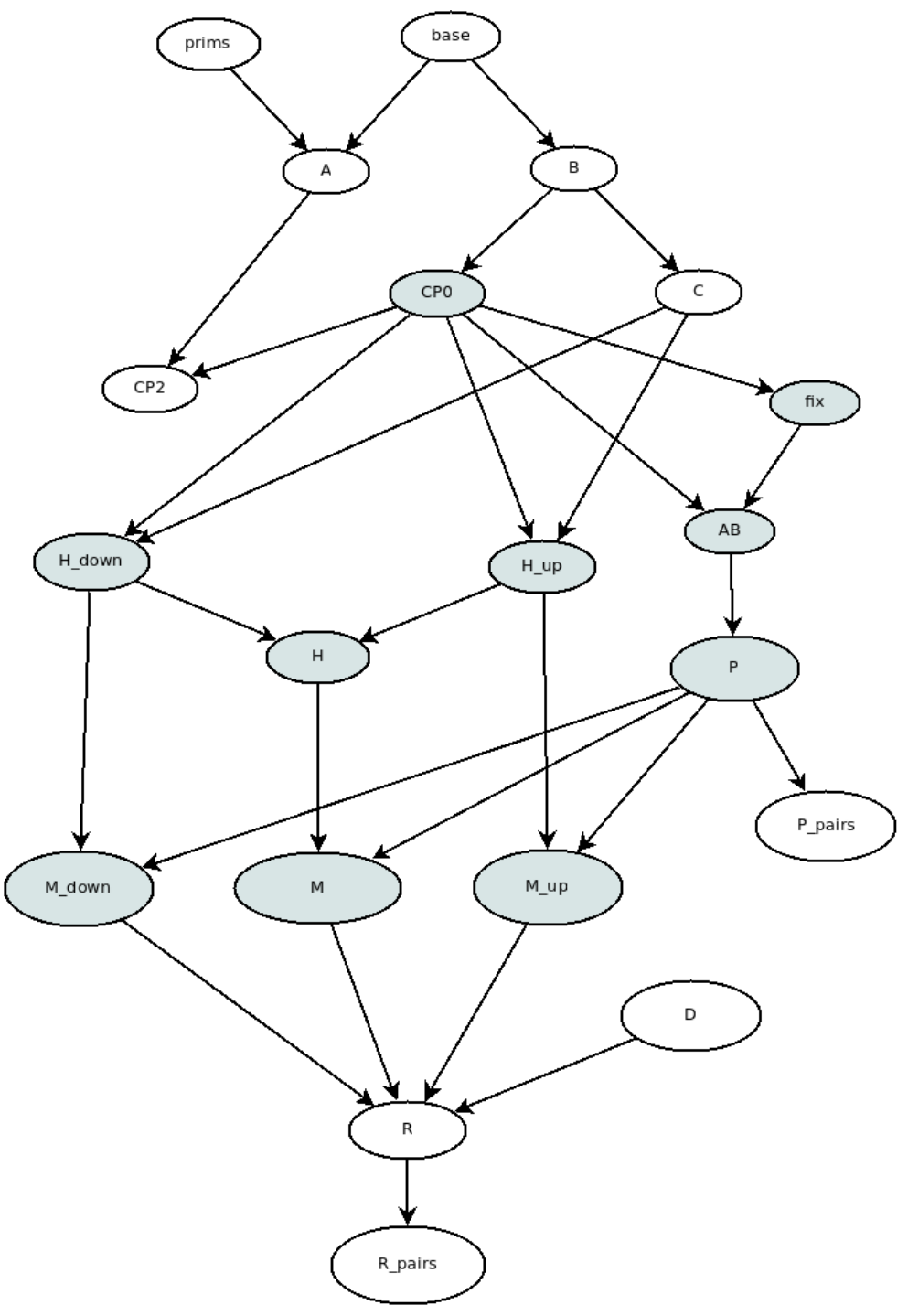

FIGURE 1. The dataflow of the calculation.

where $x \in B$. This list of lists does not stored instead it is a function of the index of the elements of $B$. The $f i x$ set contains only the indices of $B$ elements where the values are the same in $\mathrm{CPO}$ vectors. We cache the indices of $B$ where the power of $g(x)$ is not in set $f i x$. 
The $P$ denotes the set of prohibited pairs. The implementation also considers the indices. We can define $P$ as the following set.

$$
\begin{gathered}
P=\left\{(x, y) \mid x, y \in \mathrm{AB}, x_{k}=x_{k+3}, x_{k}=y_{l},(k-l) \quad \bmod 3 \neq 0,\right. \\
k \in[1 ; 4], l \in[1 ; 7]\}
\end{gathered}
$$

The $C$ matrix is only a boolean function in the implementation,

$$
C(x, y)=\left\{\begin{array}{l}
\text { true, } \quad x_{i} \leq y_{i}, i \in[1 ; 8], \\
\text { false, } \quad \text { else, }
\end{array}\right.
$$

where $x, y \in \mathrm{B}$. The H_down, $\mathrm{H}$ and $\mathrm{H}_{-}$up sets contain the elements of the set $B$,

$$
\begin{gathered}
\mathrm{H} \_ \text {up }=\left\{x \in \mathrm{CP} 0, x_{j} \neq x_{k}, C\left(x_{j}, x_{k}\right)=\text { true }, \forall j, k, j \neq k\right\}, \\
\mathrm{H} \_ \text {down }=\left\{x \in \mathrm{CP} 0, x_{j} \neq x_{k}, C\left(x_{k}, x_{j}\right)=\text { true }, \forall j, k, j \neq k\right\}, \\
\mathrm{H}=\mathrm{CP} 0 \backslash\left(\mathrm{H} \_ \text {up } \cup \mathrm{H} \text { _down }\right) .
\end{gathered}
$$

The M_down, M and M_up are the sets of pairs from $A$ and defined as the following:

$$
\begin{gathered}
\text { M_down }=\left\{(x, y) \mid x \in \mathrm{H} \_ \text {down, } y \in \mathrm{CP} 0, \exists m, t \in[1 ; 7], t \leq m,\right. \\
\left.C\left(x_{t}, y_{m}\right)=\text { true }, x_{m} \neq y_{m},(x, y) \notin \mathrm{P}\right\}, \\
\mathrm{M}=\left\{(x, y) \mid x \in \mathrm{H}, y \in \mathrm{CP} 0, \exists m, t, m^{\prime}, t^{\prime} \in[1 ; 7], m \leq t, t^{\prime} \leq m^{\prime}, C\left(x_{t}, y_{m}\right)=\text { true },\right. \\
\left.C\left(x_{t^{\prime}}, y_{m^{\prime}}\right)=\text { true }, x_{m} \neq y_{m}, x_{m^{\prime}} \neq y_{m^{\prime}},(x, y) \notin \mathrm{P}\right\}, \\
\mathrm{M} \_ \text {up }=\left\{(x, y) \mid x \in \mathrm{H} \_ \text {up }, y \in \mathrm{CP} 0, \exists m, t \in[1 ; 7], m \leq t,\right. \\
\left.C\left(x_{t}, y_{m}\right)=\text { true }, x_{m} \neq y_{m},(x, y) \notin \mathrm{P}\right\} .
\end{gathered}
$$

\section{VisUALIZATION OF THE MAP OF UNARY ALGEBRA}

For visualizing the map of unary algebra for $K=1$ we used GraphViz [1]. At the first step, we collected the elements of the map, and save them as a text file with gv extension.

Unfortunately, the default radial layout did not make possible to use those graph image as a figure. Instead, we have implemented an own graph layout calculation by using the turt le module of the Python library [4].

At the last step, we have converted the resulted layout to TikZ format [5]. We can see the results of these visualization process at the Figure 2, 3, 4, 5 and 6.

Thanks to the resulted map we have obtained eight disjunct sub-graphs. Moreover, the symmetry among the sub-graphs has revealed. The following sub-graphs are pairwise isomorph:

- $[1]_{g}$ and $[30]_{g}$,

- $[2]_{g}$ and $[6]_{g}$,

- $[7]_{g}$ and $[210]_{g}$,

- $[14]_{g}$ and $[42]_{g}$. 
pair-wise isomorphism parts. There are four components which contain proper cycle with 3 elements.

$$
\begin{aligned}
& C 19 \longrightarrow 1 \\
& \subset 570 \longrightarrow 30
\end{aligned}
$$
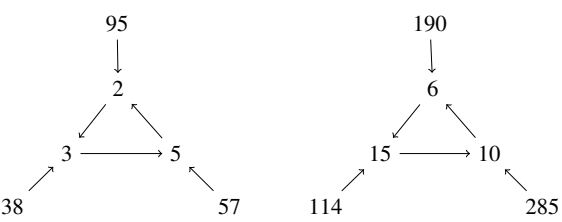

FIGURE 2. The graphs of $[1]_{g},[30]_{g},[2]_{g}$ and $[6]_{g} g$-components

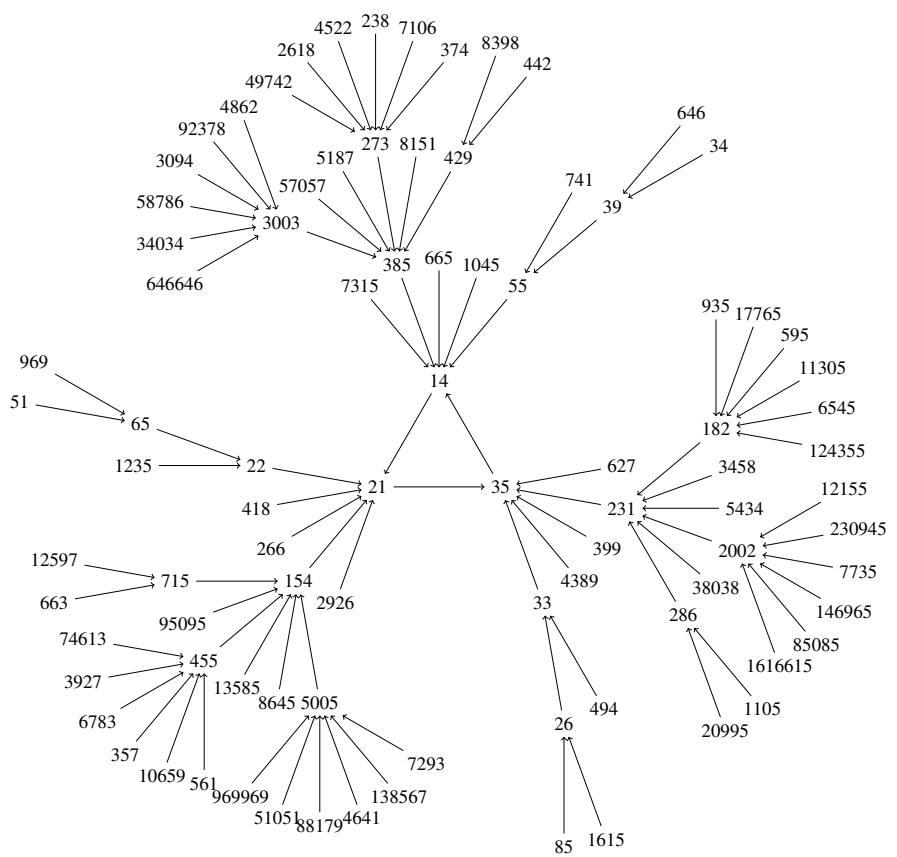

FIGURE 3. The graph of $[14]_{g} g$-component 


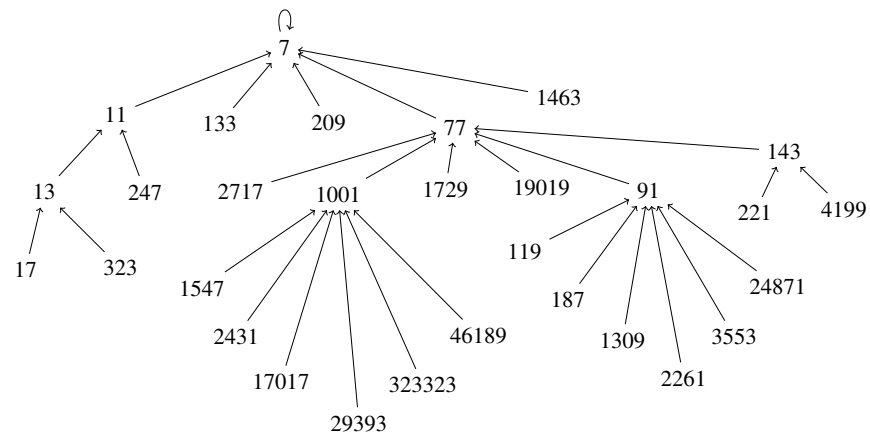

FIGURE 4 . The graph of $[7]_{g} g$-component

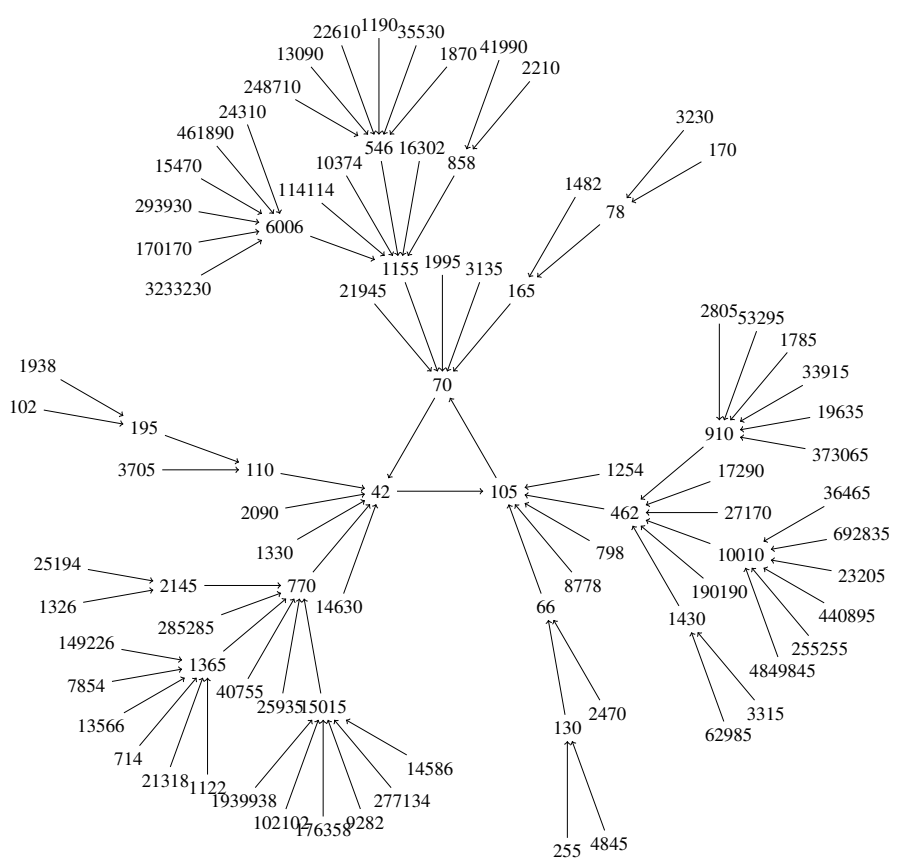

FIGURE 5. The graph of [42] $g$-component 


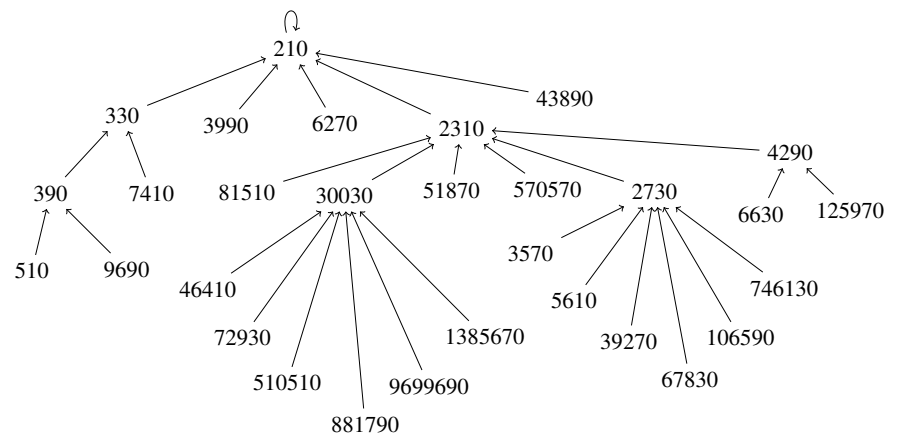

FIGURE 6 . The graph of [210 $]_{g} g$-component 


\section{REFERENCES}

[1] “Graphviz - graph visualization software,” http://graphviz.org, accessed: 2017-10-20.

[2] "Jcluster," http://freecode.com/projects/jcluster, accessed: 2017-10-20.

[3] "Magma," http://magma.maths.usyd.edu.au/magma, accessed: 2017-10-20.

[4] "Python, turtle module," https://docs.python.org/3.6/library/turtle.html, accessed: 2017-10-20.

[5] “Tikz, pgf macro package," https://www.ctan.org/pkg/pgf, accessed: 2017-10-20.

[6] S. Foldes and J. Szigeti, "Maximal compatible extensions of partial orders," Journal of the Australian Mathematical Society, vol. 81, no. 2, pp. 245-252, 2006.

[7] J. Szigeti, "On the intersection of monotonicity preserving linear extensions," Acta Mathematica Hungarica, vol. 55, no. 1-2, pp. 161-163, 1990.

[8] J. Szigeti and B. Nagy, "Linear extensions of partial orders preserving monotonicity," Order, vol. 4, no. 1, pp. 31-35, 1987.

[9] S. Szilágyi, "A characterization and the intersection of the maximal compatible extensions of a partial order," Order, vol. 25, no. 4, p. 321, 2008.

[10] E. Szpilrajn, "Sur l'extension de l'ordre partiel," Fundamenta mathematicae, vol. 16, no. 1, pp. 386-389, 1930.

Authors' addresses

Attila Házy

University of Miskolc, Institute of Mathematics, Miskolc, Hungary 3515

E-mail address: matha@uni-miskolc.hu

Péter Olajos

University of Miskolc, Institute of Mathematics, Miskolc, Hungary 3515

E-mail address: matolajeuni-miskolc.hu

\section{Imre Piller}

University of Miskolc, Institute of Mathematics, Miskolc, Hungary 3515

E-mail address: imre.piller@uni-miskolc.hu

\section{Szilvia Szilágyi}

University of Miskolc, Institute of Mathematics, Miskolc, Hungary 3515

E-mail address: matszisz@uni-miskolc.hu 